\title{
COVID19-related and all-cause mortality risk among middle-aged and older adults across the first epidemic wave of SARS- COV-2 infection: a population-based cohort study in Southern Catalonia, Spain, March- June 2020
}

Angel Vila-Corcoles ${ }^{1,2}$, Eva Satue-Gracia ${ }^{1,2}$, Angel Vila-Rovira ${ }^{2}$, Cinta de Diego-Cabanes ${ }^{1}$, Maria Jose Forcadell-Peris ${ }^{1}$, Immaculada Hospital-Guardiola', Olga Ochoa-Gondar ${ }^{1,2^{*}}$ and Josep Basora-Gallisa ${ }^{2}$

\begin{abstract}
Background: Direct and indirect COVID19-related mortality is uncertain. This study investigated all-cause and COVID19-related deaths among middle-aged and older adults during the first wave of COVID-19 pandemic period, assessing mortality risks by pre-existing socio-demographic and medical underlying conditions.

Methods: Population-based cohort study involving 79,083 individuals $\geq 50$ years-old in Tarragona (Southern Catalonia, Spain). Baseline cohort characteristics (age/sex, comorbidities and medications/vaccinations history) were established at study start (01/03/2020) and main outcomes were COVID19-related deaths (those occurred among patients with laboratory-confirmed COVID19) and all-cause deaths occurred among cohort members between 01/ 03/2020-30/06/2020. Mortality risks were assessed by Cox regression analyses.
\end{abstract}

\footnotetext{
* Correspondence: oochoa.tgn.ics@gencat.cat

'Primary Health Care Service Camp de Tarragona, Institut Catala de la Salut, Rambla Nova 124, D, $1^{\circ} \mathrm{A}, 43007$ Tarragona, Spain

${ }^{2}$ Research Universitary Institute IDIAP Jordi Gol, Barcelona, Spain
}

(c) The Author(s). 2021 Open Access This article is licensed under a Creative Commons Attribution 4.0 International License, which permits use, sharing, adaptation, distribution and reproduction in any medium or format, as long as you give appropriate credit to the original author(s) and the source, provide a link to the Creative Commons licence, and indicate if changes were made. The images or other third party material in this article are included in the article's Creative Commons licence, unless indicated otherwise in a credit line to the material. If material is not included in the article's Creative Commons licence and your intended use is not permitted by statutory regulation or exceeds the permitted use, you will need to obtain permission directly from the copyright holder. To view a copy of this licence, visit http://creativecommons.org/licenses/by/4.0/ The Creative Commons Public Domain Dedication waiver (http://creativecommons.org/publicdomain/zero/1.0/) applies to the data made available in this article, unless otherwise stated in a credit line to the data. 
Results: Cohort members were followed for 1,356,358 persons-weeks, occurring 576 all-cause deaths (124 COVID19related deaths). Of the 124 deceased patients with a laboratory-confirmed COVID19, 112 (90.3\%) died by (due to) COVID-19, while 12 (9.7\%) died with COVID-19 (but likely due to other concomitant causes). All-cause mortality rate among cohort members across study period was 42.5 deaths per 100,000 persons-week, being 22.8 among healthy/ unrelated-COVID19 subjects, 236.4 in COVID19-excluded/PCR-negative subjects, 493.7 in COVID19-compatible/PCRunperformed subjects and 4009.1 in COVID19-confirmed patients. Increasing age, sex male, nursing-home residence, cancer, neurologic, cardiac or liver disease, receiving diuretics, systemic corticosteroids, proton-pump inhibitors and benzodiazepines were associated with increased risk of all-cause mortality; conversely, receiving renin-angiotensin inhibitors and statins were associated with reduced risk. Age/years (hazard ratio [HR]: 1.08; 95\% confidence interval [Cl]: 1.06-1.10), sex male (HR: 1.82; 95\% Cl: 1.24-2.70), nursing-home residence (HR: 12.56; 95\% Cl: 8.07-19.54) and number of pre-existing comorbidities (HR: 1.14; $95 \% \mathrm{Cl}$ : 1.01-1.29) were significant predictors for COVID19-related mortality, but none specific comorbidity emerged significantly associated with an increased risk in multivariable analysis evaluating it.

Conclusion: COVID19-related deaths represented more than $20 \%$ of all-cause mortality occurred among middleaged and older adults during the first wave of the pandemic in the region. A considerable proportion (around 10 \%) of these COVID19-related deaths could be attributed to other concomitant causes. Theoretically COVID19excluded subjects (PCR-negative) suffered ten-times greater all-cause mortality than healthy/unrelated-COVID19 subjects, which points to the existence of considerable number of false negative results in earlier PCR testing and could explain part of the global excess all-cause mortality observed during the pandemic.

Keywords: Coronavirus, SARS-COV-2, COVID19, Mortality, Risk

\section{Introduction}

The new Severe Acute Respiratory Syndrome Coronavirus 2 (SARS-CoV-2) is causing the greatest infectious pandemic (COVID19) in this century [1,2]. Available publications have reported different percentages of asymptomatic or mild cases of COVID-19 but, even if they account for around 50\% [3], due to the high infectivity of the virus, the number of disease's related deaths is very high $[1,2]$. The reported mortality rates are very heterogeneous, depending on the countries (with important differences between regions, even within the same country), and also on the availability / policy of conducting diagnostic tests. Earlier studies in Lombardy (Italy) reported case fatality rates ranging between 1.6 and $18.3 \%$ [4], whereas case-fatality reported in China was $2.3 \%$ [5].

Most articles report in-hospital mortality or case fatality rates (mortality rate among confirmed cases), but it must be taken into account that infection fatality rate (which consider all infected individuals) would be lower. On the other hand, many deaths from COVID-19 occur in undiagnosed patients. Lastly, we have to keep in mind the "excess mortality" (deaths caused by other conditions, linked to a delay in care, overburden of the health system and socioeconomic determinants of health) $[6,7]$.

In Spain, a scientific-technical report of the Ministry of Health (dated November 12, 2020) informed a fatality rate of $11 \%$ in patients $\geq 50$ years (reaching $19 \%$ in the group $\geq 70$ years) [8]. However, the estimates that took into account the results of the seroprevalence study (done in May) [9] reported much lower rates [8]. According to the Statistics National Institute (INE), as compared with 2019, almost a $20 \%$ increased all-cause death occurred in Spain during 2020 (surely as consequence of COVID-19 pandemic) [10]. Similarly, in Italy an excess mortality of 99,289 deaths was reported for 2020 (from March); and in the USA, 522,368 excess deaths $(22,9 \%$ more than expected) occurred between March 1, 2020 and January 2, 2021 [11, 12]. In sum, a year after the pandemic started and more than 2 million deaths that have been attributed to the COVID-19 around the world [1,2], direct and indirect mortality due to COVID-19 is still unclear and remains controversial since case-fatality and mortality rates varying widely have been reported $[1,2,5,6,8]$.

On other hand, considering risk factors for COVID-19 mortality, most reviews agree that age and gender are well-established risk conditions for lethality in COVID19 patients [13, 14], but there is no clear consensus on the specific contribution of pre-existing conditions to the risk of mortality from the disease [15]. On this concern, knowing the relative contribution of factors related to the patient (including socio-demographic and medical aspects) to mortality is very important to identify the individuals with greater risk and guarantee a more efficient use of resources.

This study investigated COVID19-related and allcause mortality among middle-aged and older adults across the first epidemic wave of COVID-19 (March- 
June 2020) in the region of Tarragona (Southern Catalonia, Spain), assessing the possible association between previous conditions (demographic, comorbidities, chronic medications' use) and risk of death (COVID19-related and/or any cause) among the general population over 50 years (who supports the greatest burden of severe disease).

\section{Methods}

\section{Design, setting and study population}

This is a population-based retrospective cohort study (began in April 2020) involving 79,083 middle-aged and older adults living in the region of Tarragona (a residential-industrial urban area in the Mediterranean coast of Southern Catalonia). Design, setting and study population have been extensively described elsewhere, in prior articles that evaluated the incidence of COVID-19 during the first 8 and 12 weeks of epidemic period in the study area $[16,17]$.

The 79,083 cohort members were all individuals $\geq 50$ years-old affiliated to the 12 Primary Care Centres (PCCs) managed by the Catalonian Health Institut in the study area (concretely, Tarragonés, Alt Camp and Conca de Barberà counties) and represented approximately $75 \%$ of overall inhabitants in this age strata according to census data [18]. Cohort members were followed since the baseline date (01/03/2020) until the occurrence of a laboratory-confirmed COVID19, death or end of protocolised study period (30/06/2020). This report focuses on cohort members who died (by COVID-19 or any cause) in the study setting during study period. Reference laboratory and hospital for the 12 participating PCCs were the Hospital Universitari Joan XXIII and its Microbiological Service in Tarragona city. The study was approved by the Ethical Committee of the Institution (Ethics Committee IDIAP Jordi Gol, Barcelona, file 20/ 065-PCV) and was conducted in accordance with the general principles for observational studies [19].

\section{Data sources}

The pre-existing CAPAMIS Research Database, an institutional clinical database used for earlier cohort studies in the region [20], was updated and exploited as primary data source in this report. Summarily, this database collects inputs from the electronic primary care clinical records system (e-CAP) from the participant PCCs, that includes administrative and clinical data, coded according to the International Classification of Diseases 10th Revision (ICD-10). It allowed us to identify sociodemographic characteristics and pre-existing medical conditions among cohort members in order to define their baseline profile at study onset (01/03/2020).

Complementarily, we collected e-cap registries from two electronic alerts related to COVID-19's laboratory results and ICD-10 codes for COVID-19 suspicion
(B34.2, B97.29) which had been added to the e-CAP system at the beginning of the epidemic period. Later, both information sources were bonded to create the baseline research database used in this study.

Vital status was considered according to administrative data which is periodically updated in the e-CAP system. In addition, data registered during emergency visits and/ or hospital-stays, were used to complement vital status data in COVID-19 cases identified across study period. These data were transferred to a standardised form, after retrospective review of electronic records carried out by a research-trained group of family physicians and were afterwards linked with the prime research database (which contained baseline characteristics and laboratory results of 79,083 cohort members.

\section{Outcome definitions}

Primary outcome was death from any cause occurred among cohort members across study period (01/03/ 2020-30/06/2020). A COVID19-confirmed case was considered when a cohort member tested positive in SARS-COV-2 using Reverse Transcription-Polymerase Chain Reaction (RT-PCR) or other laboratory microbiological tests, according institutional guidelines [21]. In the territory, laboratory testing strategies changed throughout the study period. At first, for primary care, PCR testing (at that time with limited availability) was recommended only for patients with fever, cough or dyspnea with an epidemiological history (travel to risk areas or close contact with confirmed cases). Further, PCR testing was also recommended, if clinical suspicion, in health workers and patients admitted to hospital or in nursing-home residences (where several outbreaks took place). From May onwards, the strategy changed and PCR testing was recommended for anyone suspected of infection, and the range of symptoms expanded. By then the number of cases had decreased considerably, taking for controlled the first wave of the pandemic in Spain.

COVID19-related death was considered when the patient died from any cause within the first 30-days after the onset of the disease or at any time during hospitalstay. Considering that necropsies were not made, death by COVID-19 (likely due to COVID-19) or death with COVID-19 (likely due to other concomitant causes) were differentiated according to clinical criteria (by consensus) of research-trained group of family physician who checked hospital and primary care clinical records in each of deceased COVID-19 case.

\section{Covariates}

Baseline covariables (defined at study-start on 01/03/ 2020) were age, sex, type of residence (communitydwelling or nursing-home), vaccinations' history, previous comorbidities and chronic medications' use (see 
Appendix). They were determined by reviewing electronic clinical records system (e-CAP) which contains specially designated fields for pre-existing comorbidities, vaccinations and medications prescribed.

At the end of study period on 30/06/2020, we distinguish 4 subgroups of cohort members (according their relation with COVID-19 across study period) in order to compare their mortality rates: (i) COVID19-unrelated/ healthy individuals (without suspicion of SARS-COV-2 infection across study period); (ii) COVID19-excluded patients (With any PCR negative test, and NO positive diagnostic test, over the study period); (iii) COVID19suspected patients (With clinical suspicion but no PCR testing); (iv) COVID19-confirmed patients (with any positive PCR, or other diagnostic test if high clinical suspicion, across study period). In individuals repeatedly tested, any positive test during the study period led to consider them as COVID19-confirmed.

\section{Statistical analysis}

Mortality Rates for COVID19-related and all-cause deaths were calculated per 100,000 persons-week, considering in the denominator the sum of the persons-time contributed for each cohort member during the study period (17.4 weeks). Confidence intervals (CIs) were calculated assuming a Poisson distribution for uncommon events.

Cox regression analyses were used to calculate unadjusted, age\&sex-adjusted and multivariable-adjusted hazards ratios (HRs) and estimate the association between baseline conditions and the time to the first outcome (COVID19-related or all-cause death) occurred among cohort members throughout the study period. All above mentioned baseline covariables (judged epidemiologically relevant) were included for the multivariable-adjusted Cox models [22]. We performed a main analysis including the total study cohort and two subgroup analyses restricted to community-dwelling individuals and nursing-home residents (which are shown in supplementary Tables S1, S2, S3, S4, S5, S6, S7, S8). Statistical significance was set at $p<0.05$ (two-tailed). The analyses were performed using IBM SPSS Statistics for Windows, version 24 (IBM Corp., Armonk, N.Y., USA).

\section{Results}

The study cohort included 37,626 (47.6\%) men and $41,407(52.4 \%)$ women, with a mean age of 65.8 years (SD: 11.3). By age groups, 42,684 (54\%) were aged 5064 years and $10,386(13.1 \%)$ were aged 80 years or older. A total of 77,669 (98.2\%) were community-dwelling and 1414 (1.8\%) nursing-home residents. Cohort members were followed for a total of 1,356,358 persons-weeks $(1,335,069$ for community-dwelling and 21,289 for nursing-home residents).
Of the total 79,083 cohort members, 4084 (5.2\%) were at least PCR tested once for SARS-COV-2 infection across the study period. Overall, 3577 cohort members were classified as COVID19-excluded subjects (any time RT-PCR tested with always negative result) whereas 507 tested at least once RT-PCR positive (319 communitydwelling and 188 nursing-home residents). Additionally, 29 cohort members had a positive rapid antigen testing for SARS-COV-2 (twenty-six community-dwelling and three nursing-home residents). Therefore, a total of 536 cohort members were classified as COVID19-confirmed. Two hundred and eighty-three cohort members had a COVID19-suspected (clinically compatible COVID-19 without laboratory testing) (Table 1).

During the study period 576 all-cause deaths were observed (413 in community-dwelling and 163 in nursing-home residents), of which 124 occurred among COVID19-confirmed cases and were considered COVID19-related deaths (63 in community-dwelling and 61 in nursing-home residents). Considering these 124 COVID-19 deceased cases, 123 (99.2\%) had been diagnosed by RT-PCR and one $(0.8 \%)$ had been diagnosed by a rapid antigen test (a very old man nursing-home resident with highly compatible COVID-19 symptomatology in the context of an outbreak inside the nursinghome residence).

Concerning cause of death, 112 (90.3\%) died by (due to) COVID-19, while 12 (9.7\%) died with COVID-19 but likely due to other concomitant causes (ten cases in community dwelling and two cases in nursing-home residents). Indeed, according to physician reviewers' criteria after checking clinical records in the total 124 COVID19 deceased patients, direct cause of death could not be SARS-COV-2 infection in 12 cases (9.7\%). These twelve cases included, besides a doubtful case classified as surgical complications after ischemic colitis, three persons with advanced neoplasias (pancreas, lung and bladder), two very old patients with bronchoaspiration, two persons with severe heart failure, two very elderly convalescent after hip fracture, one advanced cirrhosis with hepatic encephalopathy and one multiple sclerosis with urinary septic shock.

Considering the total 576 cohort members who died by any cause throughout the study period, three (0.5\%)were registered during the first week, $27(4.7 \%)$ in the second, $28(4.9 \%)$ in the third, $51(8.9 \%)$ in the 4 th, $73(12.7 \%)$ in the 5th, $52(9.0 \%)$ in the 6th, $52(9.0 \%)$ in the 7 th, $54(9.4 \%)$ in the 8 th, $30(5.2 \%)$ in the 9th, 33 $(5.7 \%)$ in the 10th, $23(4.0 \%)$ in the 11th, $24(4.2 \%)$ in the 12th, $25(4.3 \%)$ in the 13th, $23(4.0 \%)$ in the 14th, 24 (4.2\%) in the 15th, $16(2.8 \%)$ and $38(6.6 \%)$ in the 17th-18th. Considering the 124 COVID19-related deaths, none occurred within the first week of the study period, one $(0.8 \%)$ occurred in the second week, one $(0.8 \%)$ in 
Table 1 Comparison of study population according to COVID19 related status at the end of study period

\begin{tabular}{|c|c|c|c|c|c|}
\hline COVID19 related status & $\begin{array}{l}\text { COVID19 } \\
\text { Nonrelated } \\
\text { (Healthy) } \\
N=74,687\end{array}$ & $\begin{array}{l}\text { COVID19 } \\
\text { Excluded } \\
\text { (PCR-) } \\
N=3577\end{array}$ & $\begin{array}{l}\text { COVID19 } \\
\text { Suspected } \\
\text { (without PCR) } \\
N=283\end{array}$ & $\begin{array}{l}\text { COVID19 } \\
\text { Confirmed } \\
\text { (PCR+) } \\
N=536\end{array}$ & $P$-value \\
\hline Characteristic & n (\%) & n (\%) & n (\%) & n (\%) & \\
\hline \multicolumn{6}{|l|}{ Sociodemographical } \\
\hline \multicolumn{6}{|l|}{ Age group } \\
\hline $50-64$ yrs & $40,799(54.6)$ & $1492(41.7)$ & $216(76.3)$ & $177(33.0)$ & $<0.001$ \\
\hline $65-79 y r s$ & $24,638(33.0)$ & $1186(33.2)$ & $52(18.4)$ & $137(25.6)$ & \\
\hline$\geq 80 \mathrm{yrs}$ & $9250(12.4)$ & $899(25.1)$ & $15(5.3)$ & $222(41.4)$ & \\
\hline \multicolumn{6}{|l|}{ Sex } \\
\hline Men & $35,575(47.6)$ & $1701(47.6)$ & $115(40.6)$ & $235(43.8)$ & 0.036 \\
\hline Women & $39,112(52.4)$ & $1876(52.4)$ & $168(59.4)$ & $301(56.2)$ & \\
\hline Community-dwelling & $73,870(98.9)$ & $3174(88.7)$ & $280(98.9)$ & $345(64.4)$ & $<0.001$ \\
\hline Nursing-home residence & $817(1.1)$ & $403(11.3)$ & $3(1.1)$ & $191(35.6)$ & \\
\hline \multicolumn{6}{|l|}{ Comorbidities } \\
\hline Neurological disease & $1935(2.6)$ & $293(8.2)$ & $10(3.5)$ & $79(14.7)$ & $<0.001$ \\
\hline Renal disease & $3982(5.3)$ & $420(11.7)$ & $9(3.2)$ & $65(12.1)$ & $<0.001$ \\
\hline Cancer & $5995(8.0)$ & $549(15.3)$ & $19(6.7)$ & $67(12.5)$ & $<0.001$ \\
\hline Rheumatic disease & $804(1.1)$ & $57(1.6)$ & $4(1.4)$ & $7(1.3)$ & 0.032 \\
\hline Inflammatory bowel disease & $497(0.7)$ & $27(0.8)$ & $2(0.7)$ & $6(1.1)$ & 0.567 \\
\hline Respiratory disease & $6592(8.8)$ & $567(15.9)$ & $33(11.7)$ & $80(14.9)$ & $<0.001$ \\
\hline Cardiac disease & $12,232(16.4)$ & $1016(28.4)$ & $34(12.0)$ & $153(28.5)$ & $<0.001$ \\
\hline Atrial fibrillation & $3317(4.4)$ & $392(11.0)$ & $7(2.5)$ & $70(13.1)$ & $<0.001$ \\
\hline Liver disease & $1338(1.8)$ & $110(3.1)$ & $6(2.1)$ & $11(2.1)$ & $<0.001$ \\
\hline Diabetes & $12,322(16.5)$ & $838(23.4)$ & $31(11.0)$ & $126(23.5)$ & $<0.001$ \\
\hline Hypertension & $32,604(43.7)$ & $1937(54.2)$ & $114(40.3)$ & $290(54.1)$ & $<0.001$ \\
\hline Hypercholesterolemia & $25,638(34.3)$ & $1398(39.1)$ & $97(34.3)$ & $181(33.8)$ & $<0.001$ \\
\hline Obesity & $20,468(27.4)$ & $1013(28.3)$ & $62(21.9)$ & $135(25.2)$ & 0.068 \\
\hline Smoking & $12,074(16.2)$ & $591(16.5)$ & $44(15.5)$ & $41(7.6)$ & $<0.001$ \\
\hline Alcoholism & $1644(2.2)$ & $134(3.7)$ & $7(2.5)$ & $11(2.1)$ & $<0.001$ \\
\hline \multicolumn{6}{|l|}{ Chronic medications use } \\
\hline Diuretics & $7586(10.2)$ & $731(20.4)$ & $25(8.8)$ & $139(25.9)$ & $<0.001$ \\
\hline Beta blockers & $8817(11.8)$ & $632(17.7)$ & $35(12.4)$ & $87(16.2)$ & $<0.001$ \\
\hline ACEls & $15,374(20.6)$ & $872(24.4)$ & $59(20.8)$ & $114(21.3)$ & $<0.001$ \\
\hline ARBs & $8317(11.1)$ & $471(13.2)$ & $27(9.5)$ & $54(10.1)$ & 0.001 \\
\hline Calcium channel blockers & $5990(8.0)$ & $420(11.7)$ & $16(5.7)$ & $64(11.9)$ & $<0.001$ \\
\hline Statins & $15,063(20.2)$ & $936(26.2)$ & $44(15.5)$ & $91(17.0)$ & $<0.001$ \\
\hline Oral anticoagulants & $3461(4.6)$ & $385(10.8)$ & $8(2.8)$ & $58(10.8)$ & $<0.001$ \\
\hline Antiplatelet drugs & $8344(11.2)$ & $676(18.9)$ & $26(9.2)$ & $108(20.1)$ & $<0.001$ \\
\hline Insulin & $2726(3.6)$ & $271(7.6)$ & $2(0.7)$ & $43(8.0)$ & $<0.001$ \\
\hline Oral antidiabetic drugs & $9855(13.2)$ & $623(17.4)$ & $22(7.8)$ & $85(15.9)$ & $<0.001$ \\
\hline Inhaled respiratory drugs & $5628(7.5)$ & $552(15.4)$ & $36(12.7)$ & $77(14.4)$ & $<0.001$ \\
\hline Antineoplastic agents & $104(0.1)$ & $12(0.3)$ & $0(0.0)$ & $0(0.0)$ & 0.017 \\
\hline Systemic corticosteroids & $1105(1.5)$ & $130(3.6)$ & $7(2.5)$ & $10(1.9)$ & $<0.001$ \\
\hline NSADs & $4079(5.5)$ & $191(5.3)$ & $30(10.6)$ & $21(3.9)$ & 0.001 \\
\hline
\end{tabular}


Table 1 Comparison of study population according to COVID19 related status at the end of study period (Continued)

\begin{tabular}{|c|c|c|c|c|c|}
\hline COVID19 related status & $\begin{array}{l}\text { COVID19 } \\
\text { Nonrelated } \\
\text { (Healthy) } \\
N=74,687\end{array}$ & $\begin{array}{l}\text { COVID19 } \\
\text { Excluded } \\
\text { (PCR-) } \\
N=3577\end{array}$ & $\begin{array}{l}\text { COVID19 } \\
\text { Suspected } \\
\text { (without PCR) } \\
N=283\end{array}$ & $\begin{array}{l}\text { COVID19 } \\
\text { Confirmed } \\
\text { (PCR+) } \\
N=536\end{array}$ & $P$-value \\
\hline Characteristic & n (\%) & n (\%) & n (\%) & n (\%) & \\
\hline Chloroquine & $154(0.2)$ & $11(0.3)$ & $2(0.7)$ & $1(0.2)$ & 0.176 \\
\hline Antihistamines & $3085(4.1)$ & $152(4.2)$ & $12(4.2)$ & $15(2.8)$ & 0.469 \\
\hline Proton-Pump Inhibitors & $16,254(21.8)$ & $1423(39.8)$ & $71(25.1)$ & $183(34.1)$ & $<0.001$ \\
\hline Benzodiazepines & $12,026(16.1)$ & $819(22.9)$ & $75(26.5)$ & $126(23.5)$ & $<0.001$ \\
\hline \multicolumn{6}{|l|}{ Vaccination's history } \\
\hline Flu vaccine in prior autumn & $20,838(27.9)$ & $1449(40.5)$ & $61(21.6)$ & $258(48.1)$ & $<0.001$ \\
\hline PPV23 & $24,189(32.4)$ & $1666(46.6)$ & $56(19.8)$ & $272(50.7)$ & $<0.001$ \\
\hline PCV13 & $1005(1.3)$ & $108(3.0)$ & $8(2.8)$ & $18(3.4)$ & $<0.001$ \\
\hline Tetanus & $47,761(63.9)$ & 2507 (70.1) & $187(66.1)$ & $344(64.2)$ & $<0.001$ \\
\hline
\end{tabular}

$P$-values were calculated by Chi-squared or Fisher's test as appropriate

the third, nine $(7.3 \%)$ in the 4 th, twenty-one $(16.9 \%)$ in the 5th, twenty-three (18.5\%) in the 6th, twenty-six $(21.0 \%)$ in the 7 th, thirteen $(10.5 \%)$ in the 8 th, seven $(5.6 \%)$ in the 9th, eight $(6.5 \%)$ in the 10th, two $(1.6 \%)$ in the 11th, four (3.2\%) in the 12th, two (1.6\%) in the 13th, four $(3.2 \%)$ in the 14 th, and one $(0.8 \%)$ in each one of the 15th, 16th and 17th weeks.

As above mentioned, of the total 536 laboratoryconfirmed COVID-19 cases, 124 patients deceased (which means a global case-fatality rate of $23.1 \%$ ). By age, case-fatality was $1.7 \%$ (3/177) in 50-64 years, $25.5 \%$ $(35 / 137)$ in $65-79$ years and $38.7 \%(86 / 222)$ in 80 years or older $(p<0.001)$. By sex, case-fatality was $26.8 \%(63 /$ $235)$ in men and $20.3 \%(61 / 301)$ in women $(p=0.075)$.
According to place of care, case-fatality rate was zero (0/ 105 for those patients exclusively managed as outpatient, $10.4 \%(5 / 48)$ for those attended in emergency rooms but not hospitalised, $28.4 \%$ (42/148) for hospitalised without ICU admission, $35.5 \%(11 / 31)$ for those admitted in the ICU, $45.2 \%$ (19/42) for those managed in social-health hospitals and $30.3 \%$ (40/165) for those patients exclusively managed in nursing-home residences. In more detail, of the 191 laboratory-confirmed COVID-19 cases occurred in nursing-home residences, four patients were referred to emergency room at general hospitals (three of them died in emergence room before hospitalisation), eighteen were hospitalised in internal medicine floor (nine of them deceased within hospital stay), one was

Table 2 Distribution of all-cause deaths according to COVID19-related status in cohort members

\begin{tabular}{lllll}
\hline $\begin{array}{l}\text { Mortality } \\
\text { Population }\end{array}$ & No. of persons & $\begin{array}{l}\text { Persons-time follow-up } \\
\text { (No. of persons-week) }\end{array}$ & No. of deaths & $\begin{array}{l}\text { Mortality Rate } \\
\text { MR (95\% Cl) }\end{array}$ \\
\hline ALL PERSONS & 79,083 & $1,356,358$ & 576 & $42.5(39.2-46.1)$ \\
$\quad$ Healthy (non-related COVID19) & 74,687 & $1,290,922$ & 290 & $22.5(20.1-25.2)$ \\
COVID19-excluded (PCR-) & 3577 & 60,825 & 145 & $238.4(201.2-282.3)$ \\
COVID19-suspected (non-PCR tested) & 283 & 1518 & 7 & $493.7(198.0-1017.0)$ \\
COVID19-confirmed (PCR+) & 536 & 3093 & 124 & $4009.1(3339.6-4810.9)$ \\
COMMUNITY-DWELING & 77,669 & $1,335,069$ & 413 & $30.9(28.0-34.1)$ \\
Healthy (non-related COVID19) & 73,870 & $1,277,550$ & 228 & $17.8(15.7-20.2)$ \\
COVID19-excluded (PCR-) & 3174 & 54,077 & $117-3$ & $216.4(180.3-259.7)$ \\
COVID19-suspected (non-PCR tested) & 280 & 1503 & $3-3$ & $199.6(41.1-582.8)$ \\
COVID19-confirmed (PCR+) & 345 & 1938 & 63 & $3250.8(2503.1-4226.0)$ \\
NURSING-HOME & 1414 & 21,289 & 163 & $765.7(653.9-896.6)$ \\
Healthy (non-related COVID19) & 817 & 13,371 & 67 & $501.1(393.4-636.4)$ \\
COVID19-excluded (PCR-) & 403 & 6747 & $33-2$ & $489.1(340.9-679.8)$ \\
COVID19-suspected (non-PCR tested) & 3 & 15 & $2-2$ & $13,333.3(1613.3-48,133.2)$ \\
COVID19-confirmed (PCR+) & 191 & 1155 & 61 & $5281.4(4066.7-6865.8)$ \\
\hline
\end{tabular}

MR denotes mortality rate, and it's calculated for 100,000 persons-week, $\mathrm{Cl}$ denotes confidence interval 
Table 3 Incidence of all-cause mortality according to baseline demographical and clinical characteristics in the total study cohort $(N=79,083)$

\begin{tabular}{|c|c|c|c|c|}
\hline \multirow[t]{2}{*}{ Characteristic } & \multirow{2}{*}{$\begin{array}{l}\text { Study } \\
\text { population } \\
(N=79,083) \\
\mathrm{n}(\%)\end{array}$} & \multicolumn{3}{|c|}{ All-cause deaths $(n=576)$} \\
\hline & & $\begin{array}{l}\text { Univariate } \\
\text { analysis } \\
\mathrm{n}(\%) p \text {-value }\end{array}$ & $\begin{array}{l}\text { Time } \\
\text { follow-up } \\
\text { (persons-week) }\end{array}$ & $\begin{array}{l}\text { Mortality rate } \\
\text { MR }(95 \% \mathrm{Cl})\end{array}$ \\
\hline \multicolumn{5}{|l|}{ Sociodemographical } \\
\hline \multicolumn{5}{|l|}{ Age } \\
\hline $50-64$ yrs & $42,684(54.0)$ & $54(9.4)<0.001$ & 734,123 & $7.4(5.5-9.8)$ \\
\hline $65-79 y r s$ & $26,013(32.9)$ & $149(25.9)$ & 447,474 & $33.3(28.1-39.4)$ \\
\hline$\geq 80 \mathrm{yrs}$ & $10,386(13.1)$ & $373(64.8)$ & 174,761 & $213.4(191.8-237.3)$ \\
\hline \multicolumn{5}{|l|}{ Sex } \\
\hline Men & $37,626(47.6)$ & $302(52.4) 0.019$ & 645,470 & $46.8(41.7-52.5)$ \\
\hline Women & $41,457(52.4)$ & $274(47.6)$ & 710,888 & $38.5(34.0-43.7)$ \\
\hline Community-dwelling & $77,676(98.2)$ & $413(71.7)<0.001$ & $1,335,069$ & $30.9(28.0-34.1)$ \\
\hline Nursing-home residence & $1407(1.8)$ & $163(28.3)$ & 21,289 & 765.7 (653.9-896.6) \\
\hline \multicolumn{5}{|l|}{ Comorbidities } \\
\hline Neurological disease & $2317(2.9)$ & $119(20.7)<0.001$ & 38,344 & $310.3(258.5-372.4)$ \\
\hline Renal disease & $4476(5.7)$ & $134(23.3)<0.001$ & 75,629 & $177.2(149.6-209.8)$ \\
\hline Cancer & $6630(8.4)$ & $168(29.2)<0.001$ & 112,481 & $149.4(127.6-174.9)$ \\
\hline Rheumatic disease & $872(1.1)$ & $10(1.7) 0.144$ & 14,897 & $67.1(32.2-123.5)$ \\
\hline Inflammatory bowel disease & $532(0.7)$ & 8 (1.4) 0.035 & 9097 & $87.9(37.9-173.2)$ \\
\hline Respiratory disease & $7272(9.2)$ & $129(22.4)<0.001$ & 123,794 & $104.2(86.8-125.0)$ \\
\hline Cardiac disease & $13,435(17.0)$ & $263(45.7)<0.001$ & 228,716 & $115.0(101.4-130.4)$ \\
\hline Atrial fibrillation & $3786(4.8)$ & $114(19.8)<0.001$ & 63,962 & $178.2(148.4-213.8)$ \\
\hline Liver disease & $1465(1.9)$ & $22(3.8)<0.001$ & 24,998 & $88.0(55.2-132.9)$ \\
\hline Diabetes & $13,317(16.8)$ & $183(31.8)<0.001$ & 227,640 & $80.4(69.3-93.3)$ \\
\hline Hypertension & $34,945(44.2)$ & $406(70.5)<0.001$ & 597,890 & $67.9(61.5-75.0)$ \\
\hline Hypercholesterolemia & $27,314(34.5)$ & 228 (39.6) 0.011 & 468,535 & $48.74(43.0-55.2)$ \\
\hline Obesity & $21,678(27.4)$ & $151(26.2) 0.518$ & 372,086 & $40.6(34.7-47.5)$ \\
\hline Smoking & $12,750(16.1)$ & $60(10.4)<0.001$ & 219,518 & $27.3(21.0-35.5)$ \\
\hline Alcoholism & $1796(2.3)$ & $17(3.0) 0.271$ & 30,774 & $55.2(32.2-88.3)$ \\
\hline \multicolumn{5}{|l|}{ Chronic medications use } \\
\hline Diuretics & $8481(10.7)$ & $238(41.3)<0.001$ & 143,357 & $166.0(146.4-188.2)$ \\
\hline Beta blockers & $9571(12.1)$ & $138(24.0)<0.001$ & 163,450 & $84.4(71.2-99.9)$ \\
\hline ACEls & $16,419(20.8)$ & $142(24.7) 0.021$ & 281,514 & $50.4(42.5-59.7)$ \\
\hline ARBs & 8869 (11.2) & $64(11.1) 0.937$ & 152,376 & $42.0(32.3-54.6)$ \\
\hline Calcium channel blockers & $6490(8.2)$ & $71(12.3)<0.001$ & 111,107 & $63.9(50.2-81.2)$ \\
\hline Statins & $16,134(20.4)$ & $123(21.4) 0.569$ & 277,237 & $44.4(37.0-53.3)$ \\
\hline Oral anticoagulants & $3912(4.9)$ & $90(15.6)<0.001$ & 66,424 & $135.5(109.6-168.0)$ \\
\hline Antiplatelet drugs & 9154 (11.6) & $173(30.0)<0.001$ & 155,957 & 110.9 (95.6-128.6) \\
\hline Insulin & $3042(3.8)$ & $60(10.4)<0.001$ & 51,741 & $116.0(89.3-150.8)$ \\
\hline Oral antidiabetic drugs & $10,585(13.4)$ & $123(21.4)<0.001$ & 181,452 & $67.8(56.5-81.4)$ \\
\hline Inhaled respiratory drugs & $6293(8.0)$ & $125(21.7)<0.001$ & 106,857 & $117.0(97.5-140.4)$ \\
\hline Antineoplastic agents & $1614(2.0)$ & 16 (2.8) 0.209 & 27,691 & $57.8(33.1-93.6)$ \\
\hline Systemic corticosteroids & $1252(1.6)$ & $56(9.7)<0.001$ & 21,030 & $266.3(205.1-346.2)$ \\
\hline NSADs & $4321(5.5)$ & $10(1.7)<0.001$ & 74,152 & $13.5(6.5-24.8)$ \\
\hline
\end{tabular}


Table 3 Incidence of all-cause mortality according to baseline demographical and clinical characteristics in the total study cohort $(N=79,083)$ (Continued)

\begin{tabular}{|c|c|c|c|c|}
\hline \multirow[t]{2}{*}{ Characteristic } & \multirow{2}{*}{$\begin{array}{l}\text { Study } \\
\text { population } \\
(N=79,083) \\
\mathrm{n}(\%)\end{array}$} & \multicolumn{3}{|c|}{ All-cause deaths $(n=576)$} \\
\hline & & $\begin{array}{l}\text { Univariate } \\
\text { analysis } \\
\mathrm{n}(\%) p \text {-value }\end{array}$ & $\begin{array}{l}\text { Time } \\
\text { follow-up } \\
\text { (persons-week) }\end{array}$ & $\begin{array}{l}\text { Mortality rate } \\
\text { MR }(95 \% \mathrm{Cl})\end{array}$ \\
\hline Chloroquine & $168(0.2)$ & $0(0.0) 0.266$ & 2867 & - \\
\hline Antihistamines & $3264(4.1)$ & $11(1.9) 0.007$ & 56,243 & $19.6(9.8-35.1)$ \\
\hline Proton-Pump Inhibitors & $17,931(22.7)$ & $312(54.2)<0.001$ & 305,566 & $102.1(91.1-114.5)$ \\
\hline Benzodiazepines & $13,046(16.5)$ & $166(28.8)<0.001$ & 222,537 & $74.6(63.7-87.4)$ \\
\hline \multicolumn{5}{|l|}{ Vaccination's history } \\
\hline Flu vaccine in prior autumn & $22,606(28.6)$ & $355(61.6)<0.001$ & 385,668 & $92.0(82.7-102.3)$ \\
\hline PPV23 & $26,183(33.1)$ & $395(68.6)<0.001$ & 447,270 & $88.3(80.0-97.5)$ \\
\hline PCV13 & $1139(1.4)$ & $24(4.2)<0.001$ & 19,272 & $124.5(79.8-185.5)$ \\
\hline Tetanus & $50,799(64.2)$ & $415(72.0)<0.001$ & 871,204 & $47.6(43.1-52.6)$ \\
\hline
\end{tabular}

$P$-values in univariate analysis were calculated by chi-squared, or Fisher's test as appropriate, comparing percentages in the study population vs percentages in allcause death cases, MR denotes mortality rates per 100.000 persons-week, Cls denotes confidence intervals for mortality rates and were calculated assuming a Poisson distribution for uncommon events

admitted in the ICU (deceasing in the ICU) and three were admitted into a social-health hospital (where one of them deceased).

Considering the total study cohort $(N=79,083)$, allcause mortality rate was 42.5 deaths per 100,000 persons-week (95\% CI: 39.2-46.1) considering the total study cohort. By residence, it was 30.9 per 100,000 persons-week (95\% CI: 28.0-34.1) in communitydwelling and 765.7 per 100,000 persons-week (95\% CI: 653.9-896.6) in nursing-home residents.

COVID19-related mortality rate was 9.1 deaths per 100,000 persons-week considering the total study cohort, being 4.7 per 100,000 persons-week among communitydwelling and 286.5 per 100,000 persons-week among nursing-home residents. Therefore, deaths from COVID-19 represented a $21.5 \%(124 / 576)$ of the overall deaths observed in the total study cohort (15.3\% [63/ 413 ] in community-dwelling vs $37.4 \%$ [61/163] in nursing-home residents).

Of the 576 all-cause deaths, 295 occurred among the 74,687 COVID19-unrelated/ healthy subjects (22.8 deaths per 100,000 persons-week), 150 deaths occurred among the 3577 COVID19-excluded/PCR-negative subjects (236.4 deaths per 100,000 persons-week), 7 among the 283 COVID19-suspected/PCR-unperformed (493.7 deaths per 100,000 persons-week) and 124 deaths occurred among the 536 COVID19-confirmed subjects (4009.1 deaths per 100,000 persons-week) (Table 2).

Table 3 reports all-cause deaths by age, sex and preexisting conditions in the total study cohort $(n=576)$. Table 4 reports it for COVID19-related deaths $(n=124)$. Tables 5 and 6 report Cox regression analyses assessing unadjusted, age\&sex-adjusted and multivariable-adjusted risks of all-cause death and COVID19-related death, respectively, in the total study cohort.

After multivariable-adjustments assessing risks of all-cause mortality in the total study cohort (Table 5), increasing age/years (HR: 1.08; 95\% CI: 1.07-1.09; $p<0.001$ ), male sex (HR: 1.47; 95\% CI: 1.24-1.79; $p<0.001$ ), nursing-home residence (HR: 1.63; 95\% CI: $1.28-2.09 ; p<0.001$ ), neurological disease (HR: 1.48; 95\% CI: $1.18-1.85 ; p=0.001$ ), cancer (HR: $2.46 ; 95 \%$ CI: 2.03-2.98; $p<0.001$ ), cardiac disease (HR: 1.29; 95\% CI: $1.06-1.56 ; p=0.009$ ), liver disease (HR: 1.76; 95\% CI: $1.14-2.73 ; p=0.012$ ), receiving diuretics (HR: 1.55; 95\% CI: $1.27-1.88$; $p<0.001)$, systemic corticosteroids (HR: 3.49; 95\% CI: 2.59-4.70; $p<0.001$ ), proton-pump inhibitors (HR: 1.25; 95\% CI: 1.03-1.51; $p=0.026$ ) and benzodiazepines (HR: 1.34; 95\% CI: $1.11-1.61 ; p=0.003)$ were associated with a significant increased risk. Conversely, receiving Angiotensin Converting Enzyme inhibitors (ACEIs) (HR: 0.73; 95\% CI: $0.60-0.88 ; p=0.001)$, Angiotensin II Receptor Blockers (ARBs) (HR: 0.59; 95\% CI: 0.46-0.77; $p<$ 0.001 ) and statins (HR: 0.72; 95\% CI: 0.59-0.88; $p=$ 0.002 ) were associated with a reduced risk. In subgroup analyses restricted to community-dwelling individuals, the results did not substantially change (see supplementary Table S3). In subgroup analysis restricted to nursing-home residents (see supplementary Table S7), history of tetanus vaccination was associated with a reduced risk (HR: 0.65; 95\% CI: 0.440.97; $p=0.036$ ).

If we consider COVID19-related mortality risk (Table 6), many comorbidities (cancer, neurological, renal, respiratory, cardiac disease, diabetes and hypertension) appeared 
Table 4 Incidence of Covid19-related mortality according to baseline demographical and clinical characteristics in the total study cohort $(N=79,083)$

\begin{tabular}{|c|c|c|c|c|}
\hline \multirow[t]{2}{*}{ Characteristic } & \multirow{2}{*}{$\begin{array}{l}\text { Study } \\
\text { population } \\
(N=79,083) \\
\mathrm{n}(\%)\end{array}$} & \multicolumn{3}{|c|}{ COVID-19 deaths $(n=124)$} \\
\hline & & $\begin{array}{l}\text { Univariate } \\
\text { analysis } \\
\mathrm{n}(\%) p \text {-value }\end{array}$ & $\begin{array}{l}\text { Time } \\
\text { follow-up } \\
\text { (persons-week) }\end{array}$ & $\begin{array}{l}\text { Mortality rate } \\
\text { MR }(95 \% \mathrm{Cl})\end{array}$ \\
\hline \multicolumn{5}{|l|}{ Sociodemographical } \\
\hline \multicolumn{5}{|l|}{ Age } \\
\hline $50-64$ yrs. & $42,684(54.0)$ & $3(2.4)<0.001$ & 734,123 & $0.4(0.1-1.2)$ \\
\hline $65-79$ yrs. & $26,013(32.9)$ & $35(28.2)$ & 447,474 & $7.8(5.4-10.8)$ \\
\hline$\geq 80 \mathrm{yrs}$ & $10,386(13.1)$ & $86(69.4)$ & 174,761 & $49.2(39.8-61.0)$ \\
\hline \multicolumn{5}{|l|}{ Sex } \\
\hline Men & $37,626(47.6)$ & $63(50.8) 0.471$ & 645,470 & $9.8(7.5-12.7)$ \\
\hline Women & $41,457(52.4)$ & $61(49.2)$ & 710,888 & $8.6(6.6-11.2)$ \\
\hline Community-dwelling & $77,676(98.2)$ & $63(50.8)<0.001$ & $1,335,069$ & $4.7(3.6-6.1)$ \\
\hline Nursing-home residence & $1407(1.8)$ & $61(49.2)$ & 21,289 & $286.5(220.6-372.5)$ \\
\hline \multicolumn{5}{|l|}{ Comorbidities } \\
\hline Neurological disease & $2317(2.9)$ & $29(23.4)<0.001$ & 38,344 & $75.6(50.7-108.9)$ \\
\hline Renal disease & $4476(5.7)$ & $23(18.5)<0.001$ & 75,629 & $30.4(19.3-45.6)$ \\
\hline Cancer & $6630(8.4)$ & $23(18.5)<0.001$ & 112,481 & $20.4(12.9-30.6)$ \\
\hline Rheumatic disease & $872(1.1)$ & $0(0.0) 0.239$ & 14,897 & - \\
\hline Inflammatory bowel disease & $532(0.7)$ & $2(1.6) 0.200$ & 9097 & $22.0(2.7-79.4)$ \\
\hline Respiratory disease & $7272(9.2)$ & $27(21.8)<0.001$ & 123,794 & $21.8(14.4-31.8)$ \\
\hline Cardiac disease & $13,435(17.0)$ & $54(43.5)<0.001$ & 228,716 & $23.6(17.5-31.2)$ \\
\hline Atrial fibrillation & $3786(4.8)$ & $23(18.5)<0.001$ & 63,962 & $36.0(22.8-54.0)$ \\
\hline Liver disease & $1465(1.9)$ & $3(2.4) 0.639$ & 24,998 & $12.0(2.5-35.0)$ \\
\hline Diabetes & $13,317(16.8)$ & $41(33.1)<0.001$ & 227,640 & $18.0(12.9-24.5)$ \\
\hline Hypertension & $34,945(44.2)$ & $79(63.7)<0.001$ & 597,890 & $13.2(10.5-16.5)$ \\
\hline Hypercholesterolemia & $27,314(34.5)$ & 48 (38.7) 0.328 & 468,535 & $10.2(7.6-13.5)$ \\
\hline Obesity & $21,678(27.4)$ & $30(24.2) 0.421$ & 372,086 & $8.1(5.5-11.6)$ \\
\hline Smoking & $12,750(16.1)$ & $11(8.9) 0.028$ & 219,518 & $5.0(2.5-9.0)$ \\
\hline Alcoholism & $1796(2.3)$ & $3(2.4) 0.912$ & 30,774 & $9.7(2.0-28.3)$ \\
\hline \multicolumn{5}{|l|}{ Chronic medications use } \\
\hline Diuretics & $8481(10.7)$ & $49(39.5)<0.001$ & 143,357 & $34.2(25.4-45.1)$ \\
\hline Beta blockers & $9571(12.1)$ & $29(23.4)<0.001$ & 163,450 & $17.7(11.9-25.5)$ \\
\hline ACEls & $16,419(20.8)$ & $32(25.8) 0.166$ & 281,514 & $11.4(7.7-16.3)$ \\
\hline ARBs & $8869(11.2)$ & $11(8.9) 0.408$ & 152,376 & $7.2(3.6-12.9)$ \\
\hline Calcium channel blockers & $6490(8.2)$ & $18(14.5) 0.010$ & 111,107 & $16.2(9.6-25.6)$ \\
\hline Statins & $16,134(20.4)$ & $26(21.0) 0.876$ & 277,237 & $9.4(6.1-13.8)$ \\
\hline Oral anticoagulants & $3912(4.9)$ & $17(13.7) 0.001$ & 66,424 & $25.6(14.9-41.0)$ \\
\hline Antiplatelet drugs & $9154(11.6)$ & $35(28.2)<0.001$ & 155,957 & $22.4(15.6-31.1)$ \\
\hline Insulin & $3042(3.8)$ & $12(9.7) 0.001$ & 51,741 & $23.2(12.0-40.6)$ \\
\hline Oral antidiabetic drugs & $10,585(13.4)$ & $33(26.6)<0.001$ & 181,452 & $18.2(12.7-25.3)$ \\
\hline Inhaled respiratory drugs & $6293(8.0)$ & $28(22.6)<0.001$ & 106,857 & $26.2(17.4-38.0)$ \\
\hline Antineoplastic agents & $1614(2.0)$ & $4(3.2) 0.350$ & 27,691 & $14.4(3.9-36.9)$ \\
\hline Systemic corticosteroids & $1252(1.6)$ & $6(4.8) 0.004$ & 21,030 & $28.5(10.5-62.1)$ \\
\hline NSADs & $4321(5.5)$ & $0(0.0) 0.007$ & 74,152 & - \\
\hline
\end{tabular}


Table 4 Incidence of Covid19-related mortality according to baseline demographical and clinical characteristics in the total study cohort $(N=79,083)$ (Continued)

\begin{tabular}{|c|c|c|c|c|}
\hline \multirow[t]{2}{*}{ Characteristic } & \multirow{2}{*}{$\begin{array}{l}\text { Study } \\
\text { population } \\
(N=79,083) \\
\mathrm{n}(\%)\end{array}$} & \multicolumn{3}{|c|}{ COVID-19 deaths $(n=124)$} \\
\hline & & $\begin{array}{l}\text { Univariate } \\
\text { analysis } \\
\mathrm{n}(\%) p \text {-value }\end{array}$ & $\begin{array}{l}\text { Time } \\
\text { follow-up } \\
\text { (persons-week) }\end{array}$ & $\begin{array}{l}\text { Mortality rate } \\
\text { MR }(95 \% \mathrm{CI})\end{array}$ \\
\hline Chloroquine & $168(0.2)$ & $0(0.0) 0.607$ & 2867 & - \\
\hline Antihistamines & $3264(4.1)$ & $0(0.0) 0.021$ & 56,243 & - \\
\hline Proton-Pump Inhibitors & $17,931(22.7)$ & $56(45.2)<0.001$ & 305,566 & $18.3(14.1-23.8)$ \\
\hline Benzodiazepines & $13,046(16.5)$ & $37(29.8)<0.001$ & 222,537 & $16.6(11.6-23.1)$ \\
\hline \multicolumn{5}{|l|}{ Vaccination's history } \\
\hline Flu vaccine in prior autumn & $22,606(28.6)$ & $88(71.0)<0.001$ & 385,668 & $22.8(18.4-28.3)$ \\
\hline PPV23 & $26,183(33.1)$ & $86(69.4)<0.001$ & 447,270 & $19.2(15.5-23.8)$ \\
\hline PCV13 & $1139(1.4)$ & $4(3.2) 0.095$ & 19,272 & $20.8(5.7-53.2)$ \\
\hline Tetanus & $50,799(64.2)$ & $82(66.1) 0.660$ & 871,204 & $9.4(7.5-11.8)$ \\
\hline
\end{tabular}

$P$-values in univariate analysis were calculated by chi-squared, or Fisher's test as appropriate, comparing percentages in the study population vs percentages in Covid19-related cases, MR denotes mortality rates per 100.000 persons-week, Cls denotes confidence intervals for mortality rates and were calculated assuming a Poisson distribution for uncommon events

associated with an increased risk in the crude analyses. However, after age\&sex-adjustment only neurological, respiratory and cardiac disease were associated with increased risk, and no specific comorbidity alone emerged significantly associated with an increased risk after multivariable-adjustment. In this analysis, increasing age/ years (HR: 1.08; 95\% CI: 1.06-1.10; $p<0.001$ ), male sex (HR: $1.82 ; 95 \% \mathrm{CI}: 1.24-2.70 ; p=0.003$ ) and nursinghome residence (HR: 12.56; 95\% CI: 8.07-19.54; $p<0.001$ ) were the strongest predictors for COVID19-related mortality. Considering chronic medications' use, while many medications were associated with an increased risk in crude analyses, none of them altered the risk of COVID19-related death in multivariable analysis (except for ARBs [HR: 0.52; 95\% CI: 0.26-1.01; $p=0.054$ ] and oral antidiabetic drugs [HR: 2.14; 95\% CI: $1.03-4.43 ; p=$ $0.042]$, that were marginally associated with reduced and increased risk, respectively).

If we consider number of pre-existing comorbidities, case-fatality rate for COVID-19 patients was 8.3\% (9/ 108) among those without comorbidities, $19.2 \%(23 / 120)$ among those with one comorbidity, 25.5\% (26/102) among those with two comorbidities, 25\% (24/96) among those with three comorbidities, $43.6 \%(24 / 55)$ among those with four comorbidities and 32.7\% (18/55) among those with five or more comorbidities $(p<$ 0.001). In analysis restricted to nursing-home COVID-19 cases $(n=191)$, case-fatality rates were $23.8 \%(5 / 21)$, $34.4 \%$ (11/32), 32.6\% (14/43), 32\% (16/50), 36\% (9/25) and $30 \%(6 / 20)$ for those with zero, one, two, three, four and five or more comorbidities, respectively $(p=0.966)$. Considering the total study cohort $(N=79,083)$, the unadjusted risk of suffering a COVID19-related death (Cox regression) increased approximately 60\% (HR: 1.60; 95\%
CI: 1.44-1.79) for each added comorbidity, whereas it was 14\% (HR: $1.14 ; 95 \%$ CI: $1.01-1.29 ; p=0.029$ ) after multivariable-adjustment by age, sex and nursing-home residence.

Supplementary Tables S1, S2, S3, S4 show subgroup analyses restricted to community-dwelling individuals and supplementary Tables S5, S6, S7, S8 show subgroup analyses restricted to nursing-home residents.

\section{Discussion}

Direct and indirect mortality due to COVID-19 is controversial $[4,5,8,13,15,23,24]$. This study investigated all-cause and COVID19-related deaths among middleaged and older adults in the region of Tarragona across the first wave of COVID-19 epidemic period (MarchJune 2020), assessing mortality risks by demographic characteristics and underlying medical conditions. Of note, the study was conducted in a region with relatively low incidence of COVID-19 as compared with other European and Spanish regions [2, 3, 8]. According to the Spanish "System for monitoring excess mortality from all causes (MoMo)", a total of 22,259 (11,969 more than expected) deaths occurred between March 13 and May 8, 2020 in Catalonia; of which, 715 (306 more than expected) were registered in Camp de Tarragona study area. This represents an excess mortality of $116 \%$ for Catalonia overall (74,8\% for Camp de Tarragona), which was higher than the corresponding to entire Spain during the same time period (65\%), lower than in Madrid (192\%) and much higher than in Galicia (19\%) [25].

In the present cohort study, ass main findings, all-cause mortality rate across study period was 42.5 deaths per 100,000 persons-week, and COVID19-related deaths represented $21.5 \%$ of overall deaths. Considering specifically 
Table 5 Cox regression analyses assessing unadjusted, age \& sex-adjusted and multivariable-adjusted risk of all-cause mortality in the total study cohort $(N=79,083)$

\begin{tabular}{|c|c|c|c|}
\hline \multirow[t]{2}{*}{ Characteristic } & \multicolumn{3}{|l|}{ All-cause deaths $(n=576)$} \\
\hline & $\begin{array}{l}\text { Unadjusted } \\
\text { HR }(95 \% \mathrm{Cl}) p \text {-value }\end{array}$ & $\begin{array}{l}\text { Age } \& \text { sex adjusted } \\
\text { HR }(95 \% \mathrm{Cl}) p \text {-value }\end{array}$ & $\begin{array}{l}\text { Multivariable } \\
\text { HR }(95 \% \mathrm{Cl}) p \text {-value }\end{array}$ \\
\hline \multicolumn{4}{|l|}{ Sociodemographical } \\
\hline Age (continuous yrs) & $1.12(1.12-1.13)<0.001$ & $1.13(1.12-1.14)<0.001$ & $1.08(1.07-1.09)<0.001$ \\
\hline Sex, women & $0.82(0.70-0.97) 0.020$ & $0.58(0.49-0.68)<0.001$ & $0.68(0.56-0.81)<0.001$ \\
\hline Nursing-home residence & $24.37(20.33-29.22)<0.001$ & $6.34(5.18-7.76)<0.001$ & $1.63(1.28-2.09)<0.001$ \\
\hline \multicolumn{4}{|l|}{ Comorbidities } \\
\hline Neurological disease & $8.90(7.28-10.89)<0.001$ & $2.55(2.06-3.15)<0.001$ & $1.48(1.18-1.85) 0.001$ \\
\hline Renal disease & $5.13(4.22-6.22)<0.001$ & $1.42(1.17-1.74) 0.001$ & $1.18(0.95-1.46) 0.130$ \\
\hline Cancer & $4.55(3.80-5.44)<0.001$ & $2.64(2.20-3.17)<0.001$ & $2.46(2.03-2.98)<0.001$ \\
\hline Rheumatic disease & $1.59(0.85-2.97) 0.146$ & $1.42(0.76-2.65) 0.276$ & $0.89(0.46-1.71) 0.717$ \\
\hline Respiratory disease & $2.87(2.36-3.49)<0.001$ & $1.75(1.43-2.13)<0.001$ & $1.26(0.96-1.66) 0.098$ \\
\hline Cardiac disease & $4.14(3.51-4.88)<0.001$ & $1.69(1.43-2.00)<0.001$ & $1.29(1.06-1.56) 0.009$ \\
\hline Atrial fibrillation & $4.98(4.06-6.11)<0.001$ & $1.50(1.22-1.85)<0.001$ & $0.87(0.63-1.19) 0.384$ \\
\hline Liver disease & $2.12(1.38-3.24) 0.001$ & $2.68(1.75-4.10)<0.001$ & $1.76(1.14-2.73) 0.012$ \\
\hline Diabetes & $2.31(1.94-2.75)<0.001$ & $1.42(1.19-1.69)<0.001$ & $1.07(0.80-1.44) 0.652$ \\
\hline Hypertension & $3.03(2.53-3.62)<0.001$ & $1.08(0.90-1.30) 0.399$ & $1.19(0.95-1.48) 0.125$ \\
\hline Obesity & $0.94(0.78-1.13) 0.513$ & $0.92(0.76-1.11) 0.274$ & $0.89(0.73-1.08) 0.219$ \\
\hline Smoking & $0.60(0.46-0.79)<0.001$ & $1.79(1.35-2.38)<0.001$ & $1.30(0.97-1.74) 0.078$ \\
\hline Alcoholism & $1.31(0.81-2.12) 0.273$ & $2.14(1.31-3.49) 0.002$ & $1.42(0.86-2.36) 0.173$ \\
\hline \multicolumn{4}{|l|}{ Chronic medications use } \\
\hline Diuretics & $5.95(5.04-7.02)<0.001$ & $2.22(1.86-2.64)<0.001$ & $1.55(1.27-1.88)<0.001$ \\
\hline Beta blockers & $2.30(1.90-2.78)<0.001$ & $1.36(1.12-1.64) 0.002$ & $1.11(0.90-1.37) 0.351$ \\
\hline ACEls & $1.25(1.03-1.51) 0.021$ & $0.73(0.60-0.88) 0.001$ & $0.64(0.52-0.79)<0.001$ \\
\hline ARBs & $0.99(0.76-1.28) 0.927$ & $0.59(0.46-0.77)<0.001$ & $0.53(0.40-0.71)<0.001$ \\
\hline Calcium channel blockers & $1.58(1.23-2.02)<0.001$ & $0.83(0.65-1.07) 0.151$ & $0.81(0.62-1.05) 0.114$ \\
\hline Statins & $1.06(0.87-1.29) 0.582$ & $0.72(0.59-0.88) 0.002$ & $0.73(0.57-0.92) 0.008$ \\
\hline Oral anticoagulants & $3.59(2.87-4.50)<0.001$ & $1.27(1.01-1.59) 0.041$ & $1.10(0.77-1.57) 0.594$ \\
\hline Antiplatelet drugs & $3.30(2.76-3.95)<0.001$ & $1.37(1.14-1.64) 0.001$ & $1.20(0.97-1.49) 0.096$ \\
\hline Insulin & $2.93(2.24-3.83)<0.001$ & $1.88(1.44-2.46)<0.001$ & $1.15(0.84-1.59) 0.376$ \\
\hline Oral antidiabetic drugs & $1.76(1.44-2.15)<0.001$ & $1.19(0.97-1.45) 0.089$ & $1.19(0.87-1.62) 0.270$ \\
\hline Inhaled respiratory drugs & $3.24(2.66-3.95)<0.001$ & $1.77(1.45-2.16)<0.001$ & $1.08(0.82-1.44) 0.575$ \\
\hline Antineoplastic agents & $1.37(0.83-2.25) 0.213$ & $1.40(0.85-2.30) 0.187$ & $0.63(0.37-1.06) 0.082$ \\
\hline Systemic corticosteroids & $6.81(5.17-8.98)<0.001$ & $4.02(3.05-5.30)<0.001$ & $3.49(2.59-4.70)<0.001$ \\
\hline NSADs & $0.31(0.16-0.57)<0.001$ & $0.52(0.28-0.98) 0.043$ & $0.65(0.35-1.23) 0.189$ \\
\hline Antihistamines & $0.45(0.25-0.82)<0.001$ & $0.52(0.29-0.95) 0.034$ & $0.58(0.32-1.05) 0.073$ \\
\hline Proton-Pump Inhibitors & $4.06(3.45-4.78)<0.001$ & $1.81(1.53-2.14)<0.001$ & $1.25(1.03-1.51) 0.026$ \\
\hline Benzodiazepines & $2.06(1.72-2.47)<0.001$ & $1.45(1.20-1.74)<0.001$ & $1.34(1.11-1.61) 0.003$ \\
\hline \multicolumn{4}{|l|}{ Vaccination's history } \\
\hline Flu vaccine in prior autumn & $4.04(3.42-4.78)<0.001$ & $1.24(1.04-1.48) 0.017$ & $1.03(0.84-1.27) 0.761$ \\
\hline PPV23 & $4.43(3.72-5.29)<0.001$ & $0.90(0.75-1.09) 0.287$ & $0.92(0.72-1.18) 0.517$ \\
\hline PCV13 & $3.01(2.00-4.53)<0.001$ & $1.81(1.20-2.72) 0.005$ & $1.13(0.74-1.73) 0.577$ \\
\hline Tetanus & $1.44(1.20-1.72)<0.001$ & $0.83(0.69-0.99) 0.042$ & $0.88(0.70-1.10) 0.263$ \\
\hline
\end{tabular}


Table 5 Cox regression analyses assessing unadjusted, age \& sex-adjusted and multivariable-adjusted risk of all-cause mortality in the total study cohort $(N=79,083)$ (Continued)

\begin{tabular}{|c|c|c|c|}
\hline \multirow[t]{2}{*}{ Characteristic } & \multicolumn{3}{|l|}{ All-cause deaths $(n=576)$} \\
\hline & $\begin{array}{l}\text { Unadjusted } \\
\text { HR }(95 \% \mathrm{Cl}) p \text {-value }\end{array}$ & $\begin{array}{l}\text { Age } \& \text { sex adjusted } \\
\text { HR }(95 \% \mathrm{Cl}) p \text {-value }\end{array}$ & $\begin{array}{l}\text { Multivariable } \\
\text { HR }(95 \% \mathrm{Cl}) p \text {-value }\end{array}$ \\
\hline \multicolumn{4}{|l|}{ COVID19-RELATED STATUS: } \\
\hline Healthy/COVID19-nonrelated & 1.00 (reference) $<0.001$ & 1.00 (reference) <0.001 & 1.00 (reference) <0.001 \\
\hline COVID19-excluded (PCR-) & $10.78(8.86-13.12)<0.001$ & $6.44(5.28-7.87)<0.001$ & $4.24(3.43-5.25)<0.001$ \\
\hline COVID19-suspected & $21.29(10.01-45.30)<0.001$ & $35.89(16.86-76.42)<0.001$ & $33.25(15.53-71.20)<0.001$ \\
\hline COVID19-confirmed (PCR+) & $181.20(144-29-227.54)<0.001$ & $74.31(58.49-94.40)<0.001$ & $45.97(35.01-60.37)<0.001$ \\
\hline
\end{tabular}

HRs denotes Hazard ratios, and were calculated for those who had the condition as compared with those who had not the condition. In multivariable analysis the HRs were adjusted for age (continuous years), sex, residence, pre-existing comorbidities/underlying conditions, chronic medications use, vaccination's history and Covid19-related status across study period, Cls denote confidence intervals

COVID19-related deaths (those occurred among patients with laboratory-confirmed COVID19), $90.3 \%$ died by COVID-19 and 9.7\% died with COVID-19 (i.e., the death could be attributed to other baseline or concomitant cause).

Increasing age, male sex, and nursing-home residence appear as the strongest predictors for both COVID19related death and all-cause death. Other pre-existing conditions may also alter mortality risk, especially some comorbidities and chronic medications' use, which emerged significantly related with all-cause mortality risk.

Of note, those cohort members who tested negative for SARS-COV-2 RT-PCR across study period (theoretically COVID-19 excluded cases) suffered approximately 10-times greater mortality (all-cause) than healthy/ COVID19-non related subjects. This fact could be expected since PCR tested subjects were older and had more comorbidities than COVID19-unrelated/healthy subjects (see Table 1), but we note that an excess risk of all-cause death by approximately 4-times (HR: 4.24; 95\% CI: 3.43-5.25) remained after multivariable-adjustments by age, sex and baseline underlying conditions (see Table 5). This data suggests that some PCR-negative subjects (theoretically COVID-19 excluded) could really suffered a COVID-19 with fatal clinical course (false negative PCR result). Indeed, this could explain, in part, the unexplained excess all-cause mortality observed in many settings during the first wave of COVID-19 pandemic. On this concern, we highlight that the reliability of RTPCR testing depends on quality of the nasopharyngeal swabs specimen, timing of collection and sensitivity of tests used [21].

Regarding excess mortality, the Spanish Statistics National Institute (INE) reported an increase in deceases in 2020 (compared to 2019) of 18.95\%; which corresponds in absolute numbers to 70,715 deaths, of which only 43,131 were attributed to COVID19 [10]. The considerably larger all-cause mortality in PCR-negative subjects observed in the present study may reflect
COVID-19 underdiagnosis and it could explain, as above mentioned, part of the excess all-cause deaths observed across the current COVID-19 pandemic.

Considering chronic medication, there is scarce data reporting the possible influence of previous use of these drugs on the risk of COVID19-related mortality. We did not observe any significant association in this sense but, interestingly, receiving statins and renin-angiotensin inhibitors were significantly associated with a lower risk of all-cause mortality in our study cohort.

We note the importance of providing adjusted data instead of crude data. Thus, while crude all-cause mortality rate was almost 25 -times greater in nursinghome (766 deaths per 100,000 persons-week) than in community-dwelling individuals (31 deaths per 100,000 persons-week), we found that after multivariable adjustments (by age/sex and pre-existing conditions) nursinghome residence only increased approximately 1.6 times the adjusted risk of all-cause mortality (HR: 1.63; 95\% CI: $1.28-2.09)$ as compared with community-dwelling (see Table 5).

In this same way, apart from increasing age and sex male, many pre-existing conditions appeared significantly associated with an increased risk of COVID19related mortality in the crude analysis (and several in the age\&sex-adjusted analysis), but none specific individual comorbidity emerged significantly associated with an increased risk after multivariable adjustment (see Table 6), "despite there was significant correlation between number of pre-existing comorbidities with the risk of COVID19-related death". This highlights the importance of maximizing adjusted data assessing event's risk in observational studies. In fact, this could explain distinct/ opposite data reported in different studies evaluating relationships between some pre-existing comorbidities/ conditions and susceptibility/risk of suffering COVID-19 infection or death $[4,5,13,15,23,24,26-29]$. To illustrate it, besides subgroup analyses, we reported all unadjusted, age\&sex-adjusted and multivariable-adjusted results here, which is a major strength in this study. 
Table 6 Cox regression analyses assessing unadjusted, age \& sex-adjusted and multivariable-adjusted risk of Covid19-related mortality in the total study cohort $(N=79,083)$

\begin{tabular}{|c|c|c|c|}
\hline \multirow[t]{2}{*}{ Characteristic } & \multicolumn{3}{|l|}{ COVID-19 deaths $(n=124)$} \\
\hline & $\begin{array}{l}\text { Unadjusted } \\
\text { HR }(95 \% \mathrm{Cl}) p \text {-value }\end{array}$ & $\begin{array}{l}\text { Age } \& \text { sex adjusted } \\
\text { HR }(95 \% \mathrm{Cl}) p \text {-value }\end{array}$ & $\begin{array}{l}\text { Multivariable } \\
\text { HR }(95 \% \mathrm{Cl}) p \text {-value }\end{array}$ \\
\hline \multicolumn{4}{|l|}{ Sociodemographical } \\
\hline Age (continuous yrs) & $1.13(1.12-1.15)<0.001$ & $1.14(1.12-1.16)<0.001$ & $1.08(1.06-1.10)<0.001$ \\
\hline Sex, women & $0.88(0.62-1.25) 0.472$ & $0.60(0.42-0.85) 0.004$ & $0.55(0.37-0.81) 0.003$ \\
\hline Nursing-home residence & $57.04(40.11-81.11)<0.001$ & $17.05(11.28-25.78)<0.001$ & $12.56(8.07-19.54)<0.001$ \\
\hline \multicolumn{4}{|l|}{ Comorbidities } \\
\hline Neurological disease & $10.30(6.79-15.61)<0.001$ & $2.74(1.77-4.23)<0.001$ & $1.35(0.86-2.14) 0.198$ \\
\hline Renal disease & $3.83(2.44-6.03)<0.001$ & $0.99(0.62-1.57) 0.952$ & $0.98(0.60-1.60) 0.943$ \\
\hline Cancer & $2.51(1.59-3.94)<0.001$ & $1.43(0.90-2.25) 0.130$ & $1.39(0.86-2.23) 0.178$ \\
\hline Rheumatic disease & $0.05(0.01-92.92) 0.433$ & NA (-) - & NA (-) - \\
\hline Respiratory disease & $2.77(1.81-4.24)<0.001$ & $1.66(1.08-2.55) 0.021$ & $1.17(0.66-2.08) 0.603$ \\
\hline Cardiac disease & $3.79(2.66-5.41)<0.001$ & $1.47(1.02-2.11) 0.039$ & $1.23(0.81-1.85) 0.333$ \\
\hline Atrial fibrillation & $4.58(2.91-7.20)<0.001$ & $1.30(0.82-2.07) 0.266$ & $1.08(0.54-2.16) 0.824$ \\
\hline Liver disease & $1.32(0.42-4.15) 0.635$ & $1.71(0.54-5.37) 0.361$ & $1.17(0.37-3.77) 0.790$ \\
\hline Diabetes & $2.45(1.68-3.56)<0.001$ & $1.50(1.03-2.19) 0.033$ & $0.85(0.41-1.77) 0.670$ \\
\hline Hypertension & $2.22(1.54-3.21)<0.001$ & $0.74(0.51-1.07) 0.110$ & $0.79(0.50-1.24) 0.297$ \\
\hline Obesity & $0.84(0.56-1.27) 0.420$ & $0.84(0.56-1.28) 0.418$ & $0.89(0.57-1.36) 0.580$ \\
\hline Smoking & $0.51(0.27-0.94) 0.031$ & $1.66(0.87-3.18) 0.126$ & $1.20(0.61-2.34) 0.600$ \\
\hline Alcoholism & $1.07(0.34-3.36) 0.911$ & $1.92(0.60-6.13) 0.272$ & $1.07(0.33-3.54) 0.910$ \\
\hline \multicolumn{4}{|l|}{ Chronic medications use } \\
\hline Diuretics & $5.49(3.83-7.87)<0.001$ & $1.88(1.29-2.74) 0.001$ & $1.48(0.97-2.25) 0.068$ \\
\hline Beta blockers & $2.22(1.47-3.37)<0.001$ & $1.30(0.86-1.97) 0.214$ & $1.24(0.78-1.98) 0.355$ \\
\hline ACEls & $1.33(0.89-1.99) 0.167$ & $0.77(0.51-1.15) 0.194$ & $0.77(0.49-1.22) 0.265$ \\
\hline ARBs & $0.77(0.42-1.43) 0.408$ & $0.46(0.25-0.85) 0.013$ & $0.52(0.26-1.01) 0.054$ \\
\hline Calcium channel blockers & $1.90(1.15-3.13) 0.012$ & $0.99(0.60-1.63) 0.970$ & $1.14(0.67-1.94) 0.633$ \\
\hline Statins & $1.04(0.67-1.59) 0.877$ & $0.73(0.47-1.12) 0.147$ & $0.91(0.54-1.52) 0.709$ \\
\hline Oral anticoagulants & $3.08(1.84-5.13)<0.001$ & $1.05(0.63-1.76) 0.854$ & $0.87(0.40-1.92) 0.734$ \\
\hline Antiplatelet drugs & $3.02(2.04-4.46)<0.001$ & $1.21(0.81-1.80) 0.356$ & $1.08(0.67-1.74) 0.750$ \\
\hline Insulin & $2.69(1.49-4.89) 0.001$ & $1.72(0.95-3.13) 0.073$ & $1.01(0.51-2.02) 0.970$ \\
\hline Oral antidiabetic drugs & $2.35(1.58-3.50)<0.001$ & $1.61(1.08-2.40) 0.019$ & $2.14(1.03-4.43) 0.042$ \\
\hline Inhaled respiratory drugs & $3.40(2.23-5.18)<0.001$ & $1.81(1.19-2.78) 0.006$ & $1.45(0.82-2.58) 0.207$ \\
\hline Antineoplastic agents & $1.60(0.59-4.33) 0.355$ & $1.65(0.61-4.47) 0.325$ & $1.55(0.55-4.39) 0.412$ \\
\hline Systemic corticosteroids & $3.19(1.41-7.25) 0.006$ & $1.84(0.81-4.19) 0.144$ & $1.88(0.80-4.41) 0.145$ \\
\hline NSADs & $0.05(0.01-1.42) 0.078$ & $N A(-)-$ & $N A(-)-$ \\
\hline Antihistamines & $0.05(0.01-2.38) 0.126$ & NA (-) - & NA (-) - \\
\hline Proton-Pump Inhibitors & $2.82(1.98-4.12)<0.001$ & $1.18(0.83-1.69) 0.361$ & $0.93(0.61-1.41) 0.716$ \\
\hline Benzodiazepines & $2.16(1.47-3.17)<0.001$ & $1.48(1.00-2.19) 0.052$ & $1.40(0.93-2.08) 0.104$ \\
\hline \multicolumn{4}{|l|}{ Vaccination's history } \\
\hline Flu vaccine in prior autumn & $6.14(4.16-9.04)<0.001$ & $1.82(1.21-2.73) 0.004$ & $1.55(0.98-2.47) 0.063$ \\
\hline PPV23 & $4.59(3.13-6.72)<0.001$ & $0.86(0.58-1.29) 0.468$ & $1.00(0.59-1.67) 0.990$ \\
\hline PCV13 & $2.30(0.85-6.23) 0.101$ & $1.37(0.51-3.71) 0.537$ & $1.15(0.41-3.21) 0.794$ \\
\hline Tetanus & $1.09(0.75-1.58) 0.659$ & $0.62(0.43-0.90) 0.013$ & $0.68(0.43-1.08) 0.103$ \\
\hline
\end{tabular}

HRs denotes Hazard ratios and were calculated for those who had the condition as compared with those who had not the condition. In multivariable analysis the HRs were adjusted for age (continuous years), sex, residence, pre-existing comorbidities/underlying conditions, chronic medications use and vaccination's history, Cls denote confidence intervals, NA Not available because zero cases in any comparison group 
Major limitations in this study were related with its retrospective design and scarce availability of PCR tests during the first weeks of study period. Indeed, since PCR testing was not always routinely performed for all clinically compatible/suspected COVID-19 patients, the number of COVID19-confirmed cases (and, consequently, the number of COVID19-related deaths) were likely underestimated. Thus, all-cause mortality (which is not influenced by the frequency of PCR testing) may be a better measure of COVID-19 pandemic impact. As another limitation, the study was conducted in a single geographical area and, logically, specific mortality data may not be directly extrapolated to other geographical regions with distinct epidemic conditions.

In order to interpret our results on COVID19-related mortality, we note that it could exist a very important difference between COVID19-suspected deaths results with COVID19-confirmed deaths (which could not be analysed in this study because necropsies were not done). Examining risk factors for COVID-19 related death, shortcomings in multivariate model analyses arise from the small sample size $(n=124)$ and, therefore, the absence of statistically significant effects does not imply the absence of clinically relevant effects.

The authors recognise these inherent limitations but note that, opposite to many papers reporting only crude COVID-19 data, the present study provides age\&sexadjusted and multivariable-adjusted data evaluating both all-cause and COVID19-related mortality risks. Importantly, the estimations may considerably vary depending on type of analyses/adjustments performed. We did subgroup analysis (nursing-home/community-dwelling) and multivariable-adjustments, but a residual confounding due to unmeasured factors (e.g., socio-economical, lifestyle, job-, healthcare-related and/or other unknown factors) may not be excluded.

\section{Conclusions}

In summary, in this population-based cohort study including 79,083 middle-aged and older adults followed across March-June 2020in the region of Tarragona, deaths from COVID-19 represented $21.5 \%$ of all-cause mortality occurred across study period. Increasing age, sex male, nursing-home residence, cancer, neurologic, cardiac or liver disease, and chronic treatment with diuretics, systemic corticosteroids, proton-pump inhibitors and benzodiazepines were associated with an increased risk of allcause mortality; conversely, receiving renin-angiotensin inhibitors and statins were associated with a reduced risk. Age/years, sex male, nursing-home residence and increasing number of pre-existing comorbidities were independent predictors for COVID19-related mortality, although no specific individual comorbidity emerged significantly associated with increased risk of COVID19-related death.
Interestingly, apart from COVID19-related deaths (approximately $10 \%$ of them could be attributed to another baseline or concomitant cause), theoretically COVID19-excluded subjects (PCR-negative) suffered 10times greater all-cause mortality than healthy/COVID19unrelated subjects. This fact points to the existence of considerable number of false negative results in earlier PCR testing [30], and may explain part of the global excess all-cause mortality observed across the pandemic.

\section{Supplementary Information}

The online version contains supplementary material available at https://doi. org/10.1186/s12889-021-11879-2.

\section{Additional file 1.}

Acknowledgements

The authors thank to Fundació Hospital Joan XXIII and Instituto de Salud Carlos III (grants files COV20/00852 and PI20/01223) their funding in publishing this paper.

\section{Authors' contributions}

AVC designed the study; AVC and ESG assessed outcomes and wrote the manuscript; ESG, CDC, MFP and IHG obtained data; ESG and AVR did statistical analyses; OOG revised the manuscript; AVC and JBG coordinated the study. The author(s) read and approved the final manuscript.

\section{Funding}

This study was supported by a grant from the Instituto de Salud Carlos III (file COV20/00852; call for the SARS-COV-2/COVID-19 disease). The funders had no role in study design, data collection and analysis, decision to publish, or preparation of the manuscript.

\section{Availability of data and materials}

Data have been obtained from the local CAPAMIS Research Database and Catalonian Health Institute Information System (e-CAP). The datasets used and/ or analysed during the current study available from the corresponding author on reasonable request.

\section{Declarations}

Ethics approval and consent to participate

The study was approved by the ethical committee of the Institution (ethic committee Institut d'Investigació en Atenció Primaria (IDIAP) Jordi Gol, Barcelona, file 20/065-PCV) and was conducted in accordance with the general principles for observational studies. Given that this is a noninterventional study, the ethics committee approved the waiver of informed consent for the 79,083 study participants under an emergency public health surveillance exception. Data were anonymized and risk of identification was null.

All methods were carried out in accordance with relevant guidelines and regulations.

\section{Consent for publication}

Not applicable.

\section{Competing interests}

The authors declare that they have no competing interests.

Received: 29 March 2021 Accepted: 20 September 2021

Published online: 06 October 2021

\section{References}

1. World Health Organisation (WHO). Coronavirus Disease (COVID19). Weekly epidemiological update- 27 January 2021. Available at: https://www.who.int/ publications/m/item/weekly-epidemiological-update\%2D\%2D-27-january-2 021. Accessed 28 Jan 2021. 
2. Johns Hopkins University of Medicine. Coronavirus Resource Center. Globa Cases. COVID-19 Dashboard by the Center for Systems Science and Engineering (CSSE) at Johns Hopkins University (JHU). Available at: https:/ coronavirus.jhu.edu/map.html. Accessed 18 Dec 2020.

3. Buitrago-Garcia D, Egli-Gany D, Counotte MJ, Hossmann S, Imeri H, Ipekci AM, et al. Occurrence and transmission potential of asymptomatic and presymptomatic SARS-CoV-2 infections: a living systematic review and meta-analysis. PLoS Med. 2020;17(9):e1003346. https://doi.org/10.1371/ journal.pmed.1003346.

4. Odone A, Delmonte D, Scognamiglio T, Signorelli C. COVID-19 deaths in Lombardy, Italy: data in context. Lancet Public Heal. 2020;5(6):e310. https:// doi.org/10.1016/S2468-2667(20)30099-2.

5. Wu C, Chen X, Cai Y, Xia J', Zhou X, Xu S, et al. Risk factors associated with acute respiratory distress syndrome and death in patients with coronavirus disease 2019 pneumonia in Wuhan, China. JAMA Intern Med. 2020;180(7): 934-43. https://doi.org/10.1001/jamainternmed.2020.0994.

6. Williamson EJ, Walker AJ, Bhaskaran K, Bacon S, Bates C, Morton CE, et al. Factors associated with COVID-19-related death using OpenSAFELY. Nature. 2020;584(7821):430-6. https://doi.org/10.1038/s41586-020-2521-4.

7. Gold JAW, Rossen LM, Ahmad FB, Sutton P, Li Z, Salvatore PP, et al. Race, ethnicity, and age trends in persons who died from COVID-19 - United States, may-august 2020. MMWR Morb Mortal Wkly Rep. 2020;69(42):151721. https://doi.org/10.15585/mmwr.mm6942e1.

8. Gobierno de España. Secretaría General de Sanidad y Consumo. Dirección General de Salud Pública, Calidad e innovación. Centro de Coordinación de Alertas y Emergencias Sanitarias. Información científica-técnica. Enfermedad por coronavirus, COVID-19 Actualización 12 de noviembre, 2020. Available at: https://www.mscbs.gob.es/profesionales/saludPublica/ccayes/alertasA ctual/nCov/documentos/TCoronavirus.pdf. Accessed 5 Dec 2020.

9. Pollán M, Pérez-Gómez B, Pastor-Barriuso R, Oteo J, Hernán MA, PérezOlmeda M, et al. Prevalence of SARS-CoV-2 in Spain (ENE-COVID): a nationwide, population-based seroepidemiological study. Lancet. 2020; 396(10250):535-44. https://doi.org/10.1016/50140-6736(20)31483-5.

10. Instituto Nacional de Estadística (INE). Estimación del número de defunciones semanales durante el brote de COVID-19. Available at: https:// www.ine.es/experimental/defunciones/experimental_defunciones.htm. Accessed 5 Dec2020.

11. Alicandro G, LA Vecchia C, Remuzzi G, Gerli A, Centanni S. Excess mortality in Italy in 2020 by sex and age groups accounting for demographic changes and temporal trends in mortality. Panminerva Med. 2021. https:// doi.org/10.23736/50031-0808.21.04397-4 [published online ahead of print, 2021 Apr 28].

12. Woolf SH, Chapman DA, Sabo RT, Zimmerman EB. Excess deaths from COVID-19 and other causes in the US, March 1, 2020, to January 2, 2021. JAMA. 2021;325(17):1786-9. https://doi.org/10.1001/jama.2021.5199 [published online ahead of print, 2021 Apr 2].

13. Figliozzi S, Masci PG, Ahmadi N, Tondi L, Koutli E, Aimo A, et al. Predictors of adverse prognosis in COVID-19: a systematic review and meta-analysis. Eur J Clin Investig. 2020;50(10):e13362. https://doi.org/10.1111/eci.13362.

14. Verity R, Okell LC, Dorigatti I, Winskill P, Whittaker C, Imai N, et al. Estimates of the severity of coronavirus disease 2019: a model-based analysis. Lancet Infect Dis. 2020;20(6):669-77. https://doi.org/10.1016/S14 73-3099(20)30243-7.

15. Ejaz H, Alsrhani A, Zafar A, Javed H, Junaid K, Abdalla AE, et al. COVID-19 and comorbidities: deleterious impact on infected patients. J Infect Public Health. 2020;13(12):1833-9. https://doi.org/10.1016/j.jph.2020.07.014.

16. Vila-Córcoles A, Ochoa-Gondar O, Torrente-Fraga C, et al. Evaluation of incidence and risk profile for suffering Covid-19 infection by underlying conditions among middle-aged and older adults in Tarragona [in Spanish]. Rev Esp Salud Pública. 2020;94:e202006065.

17. Vila-Córcoles A, Ochoa-Gondar O, Satué-Gracia EM, Torrente-Fraga C, Gomez-Bertomeu F, Vila-Rovira A, et al. Influence of prior comorbidities and chronic medications use on the risk of COVID-19 in adults: a populationbased cohort study in Tarragona, Spain. BMJ Open. 2020;10(12):e041577. https://doi.org/10.1136/bmjopen-2020-041577.

18. IDESCAT. Statistical Institute of Catalonia. Available at: https://www.idescat cat/llang=en. Accessed 20 Dec 2020.

19. World Medical Association. WMA Declaration of Helsinki - Ethical Principles for Medical Research Involving Human Subjects. Available at: https://www. wma.net/policies-post/wmadeclaration-of-helsinki-ethical-principles-formedical-research-involving-human-subjects/. Accessed 20 Dec 2020.
20. Vila-Corcoles A, Hospital-Guardiola I, Ochoa-Gondar O, de Diego C, Salsench $E$, Raga X, et al. Rationale and design of the CAPAMIS study: effectiveness of pneumococcal vaccination against community-acquired pneumonia, acute myocardial infarction and stroke. BMC Public Health. 2010;10(1):25. https:// doi.org/10.1186/1471-2458-10-25.

21. Generalitat de Catalunya. Subdirecció General de Vigilància i Resposta a Emergències de Salut Pública. Procediment d'actuació enfront de casos d'infecció pel nou coronavirus SARSCoV-2. Available at: https://canalsalut. gencat.cat/web/content/_A-Z/C/coronavirus-2019-ncov/material-divulgatiu/ procediment-actuacio-coronavirus.pdf Accessed 20 Dec 2020.

22. Hosmer DW, Lemeshow S. Applied Survival Analysis. Regression modeling of time to event data. New York: Wiley; 1999.

23. Yang J, Zheng Y, Gou X, Pu K, Chen Z, Guo Q, et al. Prevalence of comorbidities and its effects in patients infected with SARS-CoV-2: a systematic review and meta-analysis. Int J Infect Dis. 2020;94:91-5. https:// doi.org/10.1016/j.jijid.2020.03.017.

24. Ssentongo P, Ssentongo AE, Heilbrunn ES, Ba DM, Chinchilli VM. Association of cardiovascular disease and 10 other pre-existing comorbidities with COVID-19 mortality: a systematic review and meta-analysis. PLoS One. 2020; 15(8):e0238215. https://doi.org/10.1371/journal.pone.0238215.

25. Instituto de Salud Carlos III. Sistema de monitorización de la mortalidad diaria por todas la causas (MoMo). Available at: https://momo.isciii.es/public/ momo/dashboard/momo_dashboard.html\#ccaa Accessed 30 June 2021.

26. Liang WH, Guan WJ, Li CC, Li YM, Liang HR, Zhao Y, et al. Clinical characteristics and outcomes of hospitalised patients with COVID-19 treated in Hubei (epicenter) and outside Hubei (non-epicenter): a Nationwide Analysis of China. Eur Respir J. 2020;55(6):2000562. https://doi.org/10.11 83/13993003.00562-2020.

27. Grasselli G, Zangrillo A, Zanella A, Antonelli M, Cabrini L, Castelli A, et al. Baseline characteristics and outcomes of 1591 patients infected with SARSCoV-2 admitted to ICUs of the Lombardy region, Italy. JAMA. 2020;323(16): 1574-81. https://doi.org/10.1001/jama.2020.5394.

28. Zhou F, Yu T, Du R, et al. Clinical course and risk factors for mortality of adult inpatients with COVID-19 in Wuhan, China: a retrospective cohort study. Lancet. 2020;395(10229):1054-62. https://doi.org/10.1016/S0140-673 6(20)30566-3.

29. Deng G, Yin M, Chen X, Zeng F. Clinical determinants for fatality of 44,672 patients with COVID-19. CritCare. 2020;24(1):179. https://doi.org/10.1186/s13 054-020-02902-w.

30. Arévalo-Rodríguez I, Buitrago-García D, Simancas-Racines D, et al. Falsenegative results of initial RT-PCR assays for covid-19: a systematic review. PLoS One. 2020;15(12):e0242958. https://doi.org/10.1371/journal. pone.0242958,

\section{Publisher's Note}

Springer Nature remains neutral with regard to jurisdictional claims in published maps and institutional affiliations.

\section{Ready to submit your research? Choose BMC and benefit from:}

- fast, convenient online submission

- thorough peer review by experienced researchers in your field

- rapid publication on acceptance

- support for research data, including large and complex data types

- gold Open Access which fosters wider collaboration and increased citations

- maximum visibility for your research: over $100 \mathrm{M}$ website views per year

At BMC, research is always in progress.

Learn more biomedcentral.com/submissions 